\title{
Off-resonance CorrecTion OPen soUrce Software (OCTOPUS)
}

\section{DOI: $10.21105 /$ joss. 02578}

\section{Software}

- Review 七

- Repository ca

- Archive ¿

Editor: Juan Nunez-Iglesias 느 Reviewers:

- Opuolival

- Cemilljungberg

Submitted: 22 July 2020 Published: 04 March 2021

\section{License}

Authors of papers retain copyright and release the work under a Creative Commons Attribution 4.0 International License (CC BY 4.0).

\section{Marina Manso Jimeno ${ }^{1,2}$, John Thomas Vaughan Jr. ${ }^{1,2}$, and Sairam Geethanath $^{2}$}

1 Department of Biomedical Engineering, Columbia University in the City of New York, USA 2

Columbia Magnetic Resonance Research Center, Columbia University in the City of New York, USA

\section{Summary}

OCTOPUS is a Python-based software for correction of off-resonance artifacts in Magnetic Resonance (MR) images. It implements three different methods for correction of both Cartesian and non-Cartesian data: Conjugate Phase Reconstruction (CPR), frequency-segmented CPR and Multi-Frequency Interpolation(MFI). OCTOPUS is easy to integrate into other two and three-dimensional reconstruction pipelines, which makes the tool highly flexible and customizable.

\section{Statement of need}

Off-resonance is an MR artifact which occurs due to field inhomogeneities, differences in tissue susceptibilities and chemical shift (Noll et al., 1991). These phenomena can cause the phase of off-resonant spins to accumulate along the read-out direction, which can turn into blurring, geometrical distortion and degradation in the reconstructed image (Luk-Pat \& Nishimura, 2001). Images acquired using long readout trajectories and/or at high fields where the field homogeneity is lower are more prone to this problem. However, such acquisition scenarios also deliver desirable properties, such as short scanning times, gradient efficiency, motion tolerance, and better signal-to-noise ratio (Chen \& Meyer, 2008).

Multiple successful off-resonance correction methods have been reported in the literature (Schomberg, 1999). Most of them are based on Conjugate Phase Reconstruction (CPR), a method that counteracts the accumulated phase by demodulating k-space data with its conjugate (Maeda et al., 1988). Faster and more efficient implementations that the original CPR have been developed, such as frequency-segmented CPR (Noll et al., 1992) and MultiFrequency Interpolation (MFI) (Man et al., 1997). Frequency-segmented CPR reconstructs the corrected image by combining the pixels of " $\mathrm{L}$ " base images according to each pixel value on a field map. Each base image corresponds to the data demodulated at a fixed frequency, with the frequency values for each base image equally spaced within the field map frequency range. MFI works in a similar way as frequency-segmented CPR, with main differences being that it requires a smaller number of base images $(L)$ and that these images are added together into the corrected image using a set of linear coefficients derived from the field map.

One can find optimised off-resonance correction capabilities within existing packages. Examples are: SPIRiT (Lustig \& Pauly, 2010), a MATLAB-based approach for auto-calibrated parallel imaging reconstruction; Ostenson's MFI implementation for Magnetic Resonance Fingerprinting (MRF) (Ostenson et al., 2017); FUGUE, a tool for Echo-Planar Imaging (EPI) distortion correction part of the FSL library (Jenkinson et al., 2012); and the MIRT toolbox, a MATLAB-based MRI reconstruction package that offers field inhomogeneity correction using iterative reconstruction methods (Fessler et al., 2005; Sutton et al., 2003). Nylund's thesis (Nylund, 2014) also contains source MATLAB code for fs-CPR and MFI correction of spiral 
images.

All of these implementations are highly specific, defined for a particular k-space trajectory or application, and/or include a single correction method. SPIRiT is devoted to correct data acquired using parallel imaging methods; Ostenson's package only corrects MRF spiral data and implements only one correction method; and FUGUE corrects distortion solely on EPI images. These limitations typically lead researchers to adapt their data in an attempt to fit them into the available pipelines or to write their own version of the methods. Either approach results in a significant investment of time and effort and can generate isolated implementations and inconsistent results. Furthermore, most of the available packages are also MATLAB-based, which unlike Python, requires users to pay a license fee.

OCTOPUS is aimed at filling this gap in MR off-resonance correction packages. It provides Python open-source code for three fundamental methods (CPR, fs-CPR, and MFI). The implementation is independent of the application and the image acquisition scheme, easing its integration into any reconstruction pipeline. OCTOPUS can also run in the browser through Google Colab, a freely hosted Jupyter notebook environment that allows one to execute Python code in the browser. Given this feature, OCTOPUS is the first zero-footprint off-resonance correction software, meaning it doesn't require software download, installation, or configuration on a user's local machine.

\section{Functionality and limitations}

OCTOPUS is aimed at MR researchers working with long-readout or field-inhomogeneity sensitive k-space trajectories or MR acquisition methods. A short demo is provided in the next section. OCTOPUS corrects or reduces geometric distortion and/or blurring present in the images due to off-resonance effects by leveraging other Python libraries, specifically NumPy (Harris et al., 2020), SciPy (Virtanen et al., 2020), scikit-image (Walt et al., 2014), NiBabel(Brett et al., 2020), Matplotlib (Hunter, 2007), OpenCV (Itseez, 2015), Pydicom (Mason et al., 2020), and PyNUFFT(Lin, 2013-). The expected output is an image with recovered, sharper edges and undistorted shape.

Also, OCTOPUS corrects off-resonance independently of whether the trajectory used to acquire the data was Cartesian or non-Cartesian. The input of the correction methods can be either image or raw data. However, using raw data as input is more efficient and may avoid non Cartesian trajectory-dependent artifacts. OCTOPUS is also able to correct 3D multi-slice and multi-channel data by feeding it to the tool in a slice- and channel-wise manner and then applying channel combination with the user's method of choice.

Presently, the software limitations include correction restricted to data acquired in the absence of acceleration techniques, long correction times for large datasets, and degraded correction quality in the presence of highly-inhomogeneous fields. Additionally, the tool has been only tested on Cartesian, EPI, and spiral data.

\section{Short demo}

To illustrate the usage of the package, we performed in silico numerical simulations using a single-shot EPI trajectory, a single-shot spiral trajectory and a simulated field map. For these experiments we used a Shepp-Logan head phantom, which simulates a section of the skull and is widely used to test reconstruction algorithms (Shepp \& Logan, 1974). Figure 1 shows all inputs and outputs of the experiment. The steps were:

1. Forward model simulation of off-resonance effect on a $128 \times 128$ Shepp-Logan phantom and $256 \mathrm{~mm} 2$ FOV. 


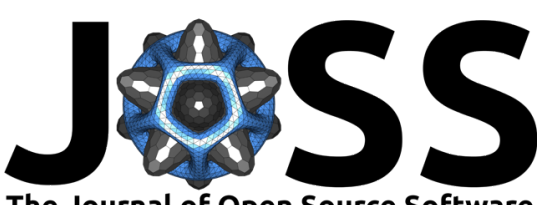

The Journal of Open Source Software

- Using single-shot EPI and spiral trajectories. Figure 1 shows simplified versions of both trajectories for visualization purposes.

- Using a simulated field map based on a blurred version of the phantom image with frequency ranges of $-/+100,-/+150$ and $-/+200 \mathrm{~Hz}$.

2. Correction of the results of the forward model with CPR, fs-CPR and MFI .

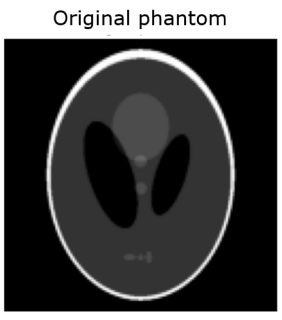

Corrupted Image CPR Correction fs-CPR Correction MFI Correction

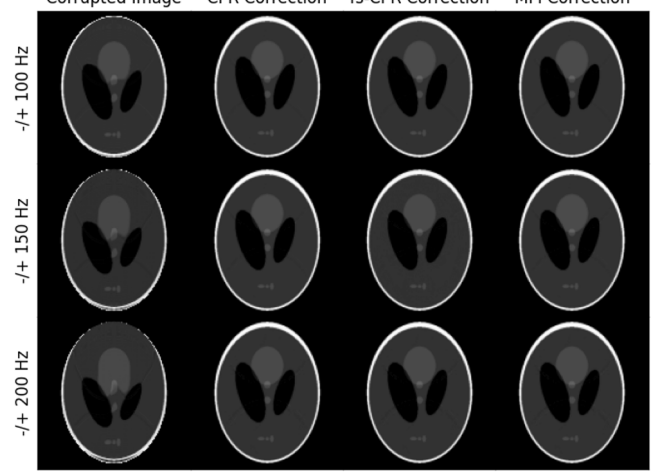

EPI

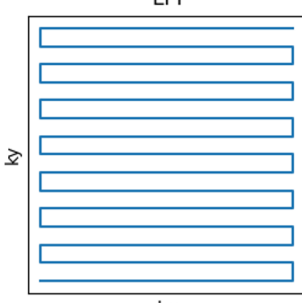

kx

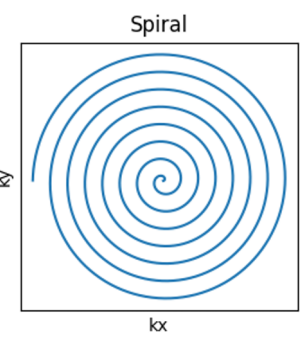

$\mathrm{kx}$

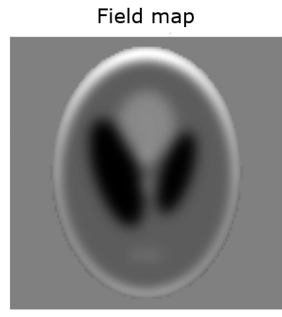

Corrupted Image CPR Correction fs-CPR Correction MFI Correction

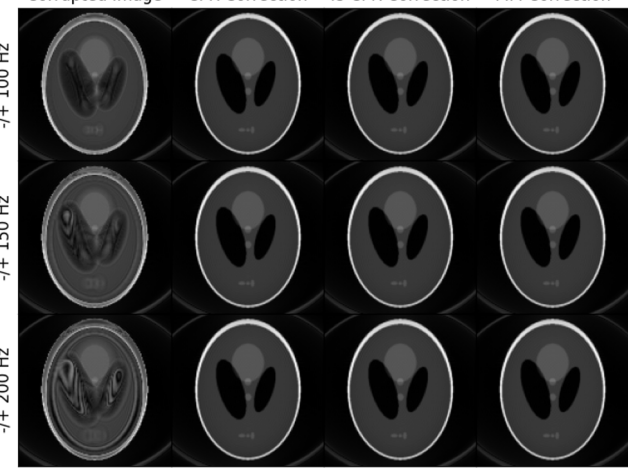

Figure 1: Top row (left-right): Shepp-Logan phantom image (128x128), Simplified single-shot EPI k-space trajectory, Simplified single-shot spiral k-space trajectory, and simulated field map $(128 \times 128)$. Bottom row (left-right): EPI experiment results and Spiral experiment results.

In both experiments, 'OCTOPUS' successfully corrected the off-resonance induced blurring and/or geometrical distortion. Note how the EPI-corrupted images show geometric distortion in the phase-encode direction while spiral corrupted images show blurred and distorted edges.

To test the effect of noise on the correction performance we introduced different levels of noise to a single-shot EPI trajectory-based simulation and measured the peak signal-to-noise ratio (pSNR) and Structural Similarity Index (SSIM). 


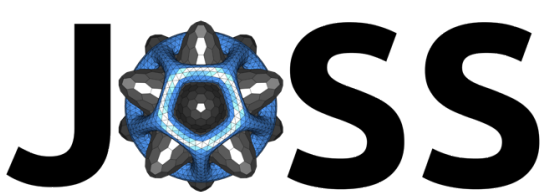

The Journal of Open Source Software

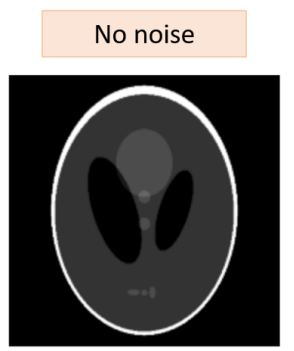

$\sigma=0.025$

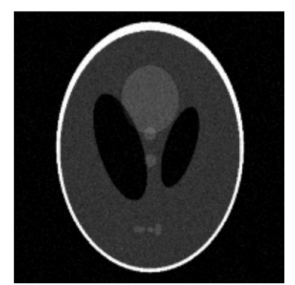

$\sigma=0.05$

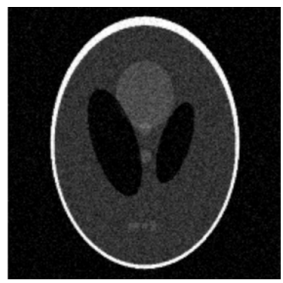

\begin{tabular}{|c|c|c|c|c|c|c|c|c|c|c|c|c|}
\hline \multirow[b]{3}{*}{ Range $(\mathrm{Hz})$} & \multicolumn{12}{|c|}{ pSNR } \\
\hline & \multicolumn{4}{|c|}{ No noise } & \multicolumn{4}{|c|}{$\sigma=0.025$} & \multicolumn{4}{|c|}{$\sigma=0.05$} \\
\hline & Corrupted & CPR & fs-CPR & MFI & Corrupted & $\mathrm{CPR}$ & fs-CPR & MFI & Corrupted & CPR & fs-CPR & MFI \\
\hline \pm 100 & 16.73 & 40.06 & 40.06 & 40.06 & 16.62 & 32.84 & 33.13 & 32.84 & 16.51 & 27.52 & 27.52 & 27.52 \\
\hline \pm 150 & 16.46 & 40.39 & 36.76 & 40.39 & 16.34 & 32.1 & 32.1 & 32.1 & 16.23 & 27.48 & 27.39 & 27.48 \\
\hline \pm 200 & 15.56 & 38.13 & 38.13 & 38.13 & 15.47 & 31.43 & 31.43 & 31.43 & 15.42 & 27.18 & 27.18 & 27.18 \\
\hline
\end{tabular}

\begin{tabular}{|c|c|c|c|c|c|c|c|c|c|c|c|c|}
\hline \multirow[b]{3}{*}{ Range $(\mathrm{Hz})$} & \multicolumn{12}{|c|}{ SSIM } \\
\hline & \multicolumn{4}{|c|}{ No noise } & \multicolumn{4}{|c|}{$\sigma=0.025$} & \multicolumn{4}{|c|}{$\sigma=0.05$} \\
\hline & Corrupted & CPR & fs-CPR & $\mathrm{MFI}$ & Corrupted & CPR & fs-CPR & MFI & Corrupted & CPR & fs-CPR & MFI \\
\hline \pm 100 & 0.77 & 1 & 1 & 1 & 0.65 & 0.86 & 0.85 & 0.86 & 0.5 & 0.64 & 0.64 & 0.64 \\
\hline \pm 150 & 0.74 & 0.99 & 0.99 & 0.99 & 0.62 & 0.86 & 0.86 & 0.86 & 0.48 & 0.64 & 0.65 & 0.64 \\
\hline \pm 200 & 0.7 & 0.99 & 0.99 & 0.99 & 0.59 & 0.86 & 0.86 & 0.86 & 0.45 & 0.65 & 0.65 & 0.65 \\
\hline
\end{tabular}

Figure 2: Effect of different noise leves on OCTOPUS correction performance measured using pSNR and SSIM.

As expected, PSNR and SSIM are reduced as the off-resonance range widens and the noise level in the original image increases. Nevertheless, in all cases, the three implemented methods improve the metrics with respect to the off-resonance corrupted image.

Finally, to demonstrate the correction capabilities in 3D multi-slice and multi-channel data, we corrected phantom images of a Stack-of-Spirals acquisition with matrix size of $72 \times 72$, $F O V=240 \mathrm{~mm} 2$ and 54 slices. The images were acquired on a Siemens 3T Prisma scanner using a 20-channel head coil. Figure 3 shows three representative slices and their off-resonance corrected versions. The regions of the images highlighted in red show improved image quality and enhaced edges. 


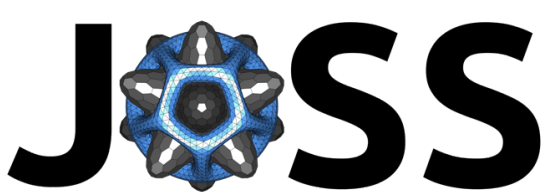

The Journal of Open Source Software



Figure 3: Off-resonance correction of three slices of a Stack-of-Spirals 3D acquisition.

\section{Acknowledgements}

This study was funded (in part) by the 'MR Technology Development Grant' and the 'Seed Grant Program for MR Studies' of the Zuckerman Mind Brain Behavior Institute at Columbia University (PI: Geethanath) and the 'Fast Functional MRI with sparse sampling and modelbased reconstruction' of the National Institute of Biomedical Imaging and Bioengineering (PI: Fessler and, supplement, sub-award to Geethanath).

\section{References}

Brett, M., Markiewicz, C. J., Hanke, M., Côté, M.-A., Cipollini, B., McCarthy, P., Cheng, C. P., Halchenko, Y. O., Cottaar, M., Ghosh, S., Larson, E., Wassermann, D., Gerhard, S., Lee, G. R., Wang, H.-T., Kastman, E., Rokem, A., Madison, C., Morency, F. C., ... freec84. (2020). Nipy/nibabel: 3.0.2 (Version 3.0.2) [Computer software]. Zenodo. https://doi.org/10.5281/zenodo.3701467

Chen, W., \& Meyer, C. H. (2008). Semiautomatic off-resonance correction in spiral imaging. Magnetic Resonance in Medicine, 59, 1212-1219. https://doi.org/10.1002/mrm.21599

Fessler, J. A., Lee, S., Olafsson, V. T., Shi, H. R., \& Noll, D. C. (2005). Toeplitz-based iterative image reconstruction for MRI with correction for magnetic field inhomogeneity. IEEE Transactions on Signal Processing, 53(9), 3393-3402. https://doi.org/10.1109/ TSP.2005.853152

Harris, C. R., Millman, K. J., Walt, S. J. van der, Gommers, R., Virtanen, P., Cournapeau, D., Wieser, E., Taylor, J., Berg, S., Smith, N. J., Kern, R., Picus, M., Hoyer, S., Kerkwijk, M. H. van, Brett, M., Haldane, A., Fernández del Río, J., Wiebe, M., Peterson, P., 
... Oliphant, T. E. (2020). Array programming with NumPy. Nature, 585, 357-362. https://doi.org/10.1038/s41586-020-2649-2

Hunter, J. D. (2007). Matplotlib: A 2D graphics environment. Computing in Science \& Engineering, 9(3), 90-95. https://doi.org/10.1109/MCSE.2007.55

Itseez. (2015). Open source computer vision library. https://github.com/itseez/opencv.

Jenkinson, M., Beckmann, C. F., Behrens, T. E. J., Woolrich, M. W., \& Smith, S. M. (2012). Review FSL. Neurolmage, 62(2), 782-790. https://doi.org/10.1016/j. neuroimage.2011. 09.015

Lin, J.-M. (2013-). Pynufft: Python non-uniform fast Fourier transform. https://github. com/jyhmiinlin/pynufft

Luk-Pat, G. T., \& Nishimura, D. G. (2001). Reducing off-resonance distortion by echo-time interpolation. Magnetic Resonance in Medicine, 45(2), 269-276. https://doi.org/10. 1002/1522-2594(200102)45:2\%3C269::AID-MRM1036\%3E3.0.CO;2-5

Lustig, M., \& Pauly, J. M. (2010). SPIRiT: Iterative self-consistent parallel imaging reconstruction from arbitrary k-space. Magnetic Resonance in Medicine, 64(2), 457-471. https://doi.org/10.1002/mrm.22428

Maeda, A., Sano, K., \& Yokoyama, T. (1988). Reconstruction by Weighted Correlation for MRI with Time-Varying Gradients. IEEE Transactions on Medical Imaging, 7(1), 26-31. https://doi.org/10.1109/42.3926

Man, L. C., Pauly, J. M., \& Macovski, A. (1997). Multifrequency interpolation for fast offresonance correction. Magnetic Resonance in Medicine, 37(5), 785-792. https://doi.org/ $10.1002 / \mathrm{mrm} .1910370523$

Mason, D., scaramallion, rhaxton, mrbean-bremen, Suever, J., Vanessasaurus, Lemaitre, G., Orfanos, D. P., Panchal, A., Rothberg, A., Massich, J., Kerns, J., Golen, K. van, Robitaille, T., moloney, Shun-Shin, M., pawelzajdel, Mattes, M., Conrad, B., ... Bryant, C. (2020). Pydicom/pydicom: Pydicom 2.0.0 (Version v2.0.0) [Computer software]. Zenodo. https: //doi.org/10.5281/zenodo.3891702

Noll, D. C., Meyer, C. H., Pauly, J. M., Nishimura, D. G., \& Macovski, A. (1991). A Homogeneity Correction Method for Magnetic Resonance Imaging with Time-Vaiying Gradients. IEEE Transactions on Medical Imaging, 10(4), 629-637. https://doi.org/10.1109/ 42.108599

Noll, D. C., Pauly, J. M., Meyer, C. H., Nishimura, D. G., \& Macovskj, A. (1992). Deblurring for non-2D fourier transform magnetic resonance imaging. Magnetic Resonance in Medicine, 25(2), 319-333. https://doi.org/10.1002/mrm.1910250210

Nylund, A. (2014). Off-resonance correction for magnetic resonance imaging with spiral trajectories (p. 43) [PhD thesis]. KTH Royal Institute of Technology.

Ostenson, J., Robison, R. K., Zwart, N. R., \& Welch, E. B. (2017). Multi-frequency interpolation in spiral magnetic resonance fingerprinting for correction of off-resonance blurring. Magnetic Resonance Imaging, 41, 63-72. https://doi.org/10.1016/j.mri.2017.07.004

Schomberg, H. (1999). Off-resonance correction of MR images. IEEE Transactions on Medical Imaging, 18(6), 481-495. https://doi.org/10.1109/42.781014

Shepp, L. A., \& Logan, B. F. (1974). FOURIER RECONSTRUCTION OF A HEAD SECTION. IEEE Transactions on Nuclear Science, 21, 21-43. https://doi.org/10.1109/tns. 1974.6499235

Sutton, B. P., Noll, D. C., \& Fessler, J. A. (2003). Fast, iterative image reconstruction for $\mathrm{MRI}$ in the presence of field inhomogeneities. IEEE Transactions on Medical Imaging, 22(2), 178-188. https://doi.org/10.1109/TMI.2002.808360 
Virtanen, P., Gommers, R., Oliphant, T. E., Haberland, M., Reddy, T., Cournapeau, D., Burovski, E., Peterson, P., Weckesser, W., Bright, J., van der Walt, S. J., Brett, M., Wilson, J., Millman, K. J., Mayorov, N., Nelson, A. R. J., Jones, E., Kern, R., Larson, E., ... SciPy 1.0 Contributors. (2020). SciPy 1.0: Fundamental Algorithms for Scientific Computing in Python. Nature Methods, 17, 261-272. https://doi.org/10.1038/ s41592-019-0686-2

Walt, S. van der, Schönberger, J. L., Nunez-Iglesias, J., Boulogne, F., Warner, J. D., Yager, N., Gouillart, E., Yu, T., \& contributors, T. scikit-image. (2014). scikit-image: image processing in $\{$ P $\}$ ython. PeerJ, 2, e453. https://doi.org/10.7717/peerj.453 